Moroccan J. of Pure and Appl. Anal. (MJPAA)

Volume 4(2), 2018, Pages 171-188

ISSN: Online 2351-8227 - Print 2605-6364

DOI 10.1515/mjpaa-2018-0014

\title{
Existence of Solutions for Some Nonlinear Elliptic Anisotropic Unilateral Problems with Lower Order Terms
}

\author{
Youssef Akdim ${ }^{1, a}$, Chakir Allalou ${ }^{2, b}$ ANd Abdelhafid Salmani $3, c$
}

AвstRAct. In this paper, we prove the existence of entropy solutions for anisotropic elliptic unilateral problem associated to the equations of the form

$$
-\sum_{i=1}^{N} \partial_{i} a_{i}(x, u, \nabla u)-\sum_{i=1}^{N} \partial_{i} \phi_{i}(u)=f,
$$

where the right hand side $f$ belongs to $L^{1}(\Omega)$. The operator $-\sum_{i=1}^{N} \partial_{i} a_{i}(x, u, \nabla u)$ is a Leray-Lions anisotropic operator and $\phi_{i} \in C^{0}(\mathbb{R}, \mathbb{R})$.

2010 Mathematics Subject Classification. Primary: 35J60, 35J87, 35J66.

Key words and phrases. Entropy solutions, Anisotropic elliptic equations, Anisotropic Sobolev space.

\section{Introduction}

Let $\Omega$ be a bounded open subset of $\mathbb{R}^{N}(N \geq 2)$ with smooth boundary and let $1<p_{1}, \ldots, p_{N}<+\infty$ be a $N$ real numbers and $\vec{p}=\left(p_{1}, \ldots, p_{N}\right)$. We consider the obstacle problem associated with the following elliptic equations

$$
\left\{\begin{array}{l}
A u-\operatorname{div} \phi(u)=f \text { in } \Omega \\
u=0 \text { on } \partial \Omega,
\end{array}\right.
$$

Received February 25, 2019 - Accepted April 18, 2019.

(C) The Author(s) 2017. This article is published with open access by Sidi Mohamed Ben Abdallah University.

${ }^{1}$ Department of Mathematics, Laboratory LAMA Faculty of Sciences, Dhar-Mahrez, Fez, Morocco.

${ }^{2}$ Sultan Moulay slimane University Laboratory LMACS,FST Beni-Mellal, Morocco.

${ }^{3}$ Laboratory LSI, Polydisciplinary Faculty, Taza.

a e-mail: akdimyoussef@yahoo.fr

${ }^{b}$ e-mail: chakir.allalou@yahoo.fr

ce-mail:slmnhfd@gmail.com. 
where $A$ is a Leray-Lions operator from anisotropic space $W_{0}^{1, \vec{p}}(\Omega)$ into its dual $W^{-1, p^{\prime}}(\Omega)$ defined by $A u=$ $-\operatorname{div} a(x, u, \nabla u)$ and $\phi=\left(\phi_{1}, \ldots, \phi_{N}\right)$ belongs to $C^{0}(\mathbb{R}, \mathbb{R})^{N}$. As regards the second member, we assume that the datum $f$ belongs to $L^{1}(\Omega)$.

In recent years an increasing interest has turned towards anisotropic elliptic and parabolic equations. A special interest in the study of such equations is motivated by their applications to the mathematical modeling of physical and mechanical processes in anisotropic continuous medium. We refer to the recent works $[4,5,21]$ where it is possible to find some references.

In $[1,7,13]$ the authors proved the existence of the solutions for some unilateral nonlinear elliptic problem in the classical Sobolev space $W_{0}^{1, p}(\Omega)$ and in the Orlicz spaces with $f \in L^{1}(\Omega)+W_{0}^{1, \overrightarrow{p^{\prime}}}(\Omega)$. L. Boccardo in [12] proved the existence of solutions of some nonlinear Dirichlet problem in $\mathrm{L}^{1}$ involving lower order terms in divergence form.

Boccardo et al. in [11] studied the existence of weak solutions for nonlinear elliptic problem (1.1) with $A u=$ $-\sum_{i=1}^{N} \frac{\partial}{\partial x_{i}}\left(\left.\left|\frac{\partial u}{\partial x_{i}}\right|\right|_{i}-2 \frac{\partial u}{\partial x_{i}}\right), \phi_{i}(u)=0$ for $i=1, \ldots, N$ and the right-hand side is a bounded Radon measure on $\Omega$. In the case where $A u=-\sum_{i=1}^{N} \frac{\partial}{\partial x_{i}} a_{i}\left(x, \frac{\partial u}{\partial x_{i}}\right), \quad \phi_{i}(u)=0$ for $i=1, \ldots, N$ and the right hand side $f=\left(f_{1}, \ldots, f_{m}\right)^{\top}$ is vector-valued Radon measure on $\Omega$ of finite mass, existence solutions of (1.1) is proved by Bendahmane et al. in [5]. We cite some papers that have dealt with the equation (1.1) or similar problems, see $[4,5,14,15,16,21]$. Note that in the isotropic case, there are large works in the direction of problem (1.2) can be found in [3, 6, 7, 8, 23].

The objective of our article is to study the anisotropic unilateral nonlinear elliptic problem associated with the nonlinear problem (1.1). More precisely, we prove the existence of entropy solutions for the following unilateral anisotropic problem.

$$
\left\{\begin{array}{l}
u \geq \psi \text { a.e. in } \Omega, \\
T_{k}(u) \in W_{0}^{1, \vec{p}}(\Omega) \quad \forall k>0, \\
\sum_{i=1}^{N} \int_{\Omega} a_{i}(x, u, \nabla u) \partial_{i} T_{k}(u-v) d x+\sum_{i=1}^{N} \int_{\Omega} \phi_{i}(u) \partial_{i} T_{k}(u-v) d x \leq \int_{\Omega} f T_{k}(u-v) d x, \\
\forall v \in K_{\psi} \cap L^{\infty}(\Omega),
\end{array}\right.
$$

where $K_{\psi}=\left\{u \in W_{0}^{1, \vec{p}}(\Omega), u \geq \psi\right.$ a.e. in $\left.\Omega\right\}$ with $\psi$ is a measurable function on $\Omega$ such that $\psi^{+} \in W_{0}^{1, \vec{p}}(\Omega) \cap$ $L^{\infty}(\Omega)$ and $T_{k}$ is the usual truncation function. Note that the existence result is proved by assuming only $\phi$ is continuous function. If we take $\psi=-\infty$, we obtain the existence results of problem (1.2) in the case of equation. The integrals in (1.2) are well defined: Indeed under the condition (3.2), the function $a_{i}(x, u, \nabla u)$ is belongs to $L^{p_{i}^{\prime}}(\Omega)$ and since $\partial_{i} T_{k}(u-v)$ is belongs to $L^{p_{i}}(\Omega)$, the first integral in the left hand in (1.2) is well defined. For the second integral in the left hand in (1.2), since $\phi_{i}(u) \partial_{i} T_{k}(u-v)=0$ on $\left\{|u|>\|v\|_{\infty}+k\right\}$ and $\phi_{i} \in C^{0}(\mathbb{R}, \mathbb{R})$, $\phi_{i}(u)$ is bounded in $\left\{|u| \leq\|v\|_{\infty}+k\right\}$, then the second integral is well defined. Moreover since $f \in L^{1}(\Omega)$ and $T_{k}(u-v) \in L^{\infty}(\Omega)$, the integral in the right hand is well defined.

Since the function $\phi_{i}(u)$ does not belong to $L_{l o c}^{1}(\Omega)$ in general, the problem (1.1) does not admit weak solutions. To overcome this difficulty, we use the entropy solutions in this work which introduced for the first time by Bnilan et al. in [8]

This paper is organized as follows: Section 2 is devoted to introduce some preliminary results including a brief discussion on the anisotropic Sobolev spaces. Section 3 is devoted to give some important Lemmas. Section 4 contains the main result. Section 5 will be devoted to show the principal proposition concerning the existence of solutions for approximate problems.

\section{Preliminaries}

Let $\Omega$ be a bounded open subset of $\mathbb{R}^{N}(N \geq 2)$ with smooth boundary and let $1<p_{1}, \ldots, p_{N}<\infty$ be $\mathrm{N}$ real numbers, $p^{+}=\max \left\{p_{1}, \ldots, p_{N}\right\}, p^{-}=\min \left\{p_{1}, \ldots, p_{N}\right\}$ and $\vec{p}=\left(p_{1}, \ldots, p_{N}\right)$. We denote $\partial_{i}=\frac{\partial}{\partial x_{i}}$. 
The anisotropic Sobolev space (see [22])

$$
W^{1, \vec{p}}(\Omega)=\left\{u \in W^{1,1}(\Omega), \partial_{i} u \in L^{p_{i}}(\Omega), i=1,2, \ldots, N\right\}
$$

is a Banach space with respect to norm

$$
\|u\|_{W^{1, \vec{p}}(\Omega)}=\|u\|_{L^{1}(\Omega)}+\sum_{i=1}^{N}\left\|\partial_{i} u\right\|_{L^{p_{i}(\Omega)}} .
$$

The space $W_{0}^{1, \vec{p}}(\Omega)$ is the closure of $C_{0}^{\infty}(\Omega)$ with respect to this norm. Let us recall the Sobolev type inequalities, for $u \in W_{0}^{1, \vec{p}}(\Omega)$, there exists a constant and $C$ (see [22]) such that

$$
\|u\|_{L^{q}(\Omega)} \leq C_{s} \prod_{i=1}^{N}\left\|\frac{\partial u}{\partial x_{i}}\right\|_{L^{p_{i}}(\Omega)^{\prime}}^{\frac{1}{N}}
$$

where $q=\bar{p}^{*}=\frac{N \bar{p}}{N-\bar{p}}$ if $\quad \bar{p}<N$ or $q \in[1,+\infty[\quad$ if $\quad \bar{p} \geq N$, which implies by (2.2)

$$
\|u\|_{L^{q}(\Omega)} \leq \frac{C_{s}}{N} \sum_{i=1}^{N}\left\|\frac{\partial u}{\partial x_{i}}\right\|_{L^{p_{i}(\Omega)}}
$$

When $\bar{p}<N$, by (2.3), we have the continuous embedding of $W_{0}^{1, \vec{p}}(\Omega)$ into $L^{q}(\Omega)$ for every $q \in\left[1, \bar{p}^{*}\right]$. The space $W_{0}^{1, \vec{p}}(\Omega)$ is separable and reflexive Banach space which satisfies the continuous imbedding $W_{0}^{1, \vec{p}}(\Omega) \hookrightarrow$ $W_{0}^{1, p_{-}}(\Omega)$ and its dual $\left(W_{0}^{1, \vec{p}}(\Omega)\right)^{\prime}$ is denoted by $W^{-1, \overrightarrow{p^{\prime}}}(\Omega)$.

Remark 2.1. As a consequence of the Sobolev imbedding and the continuous imbedding $W_{0}^{1, \vec{p}}(\Omega) \hookrightarrow W_{0}^{1, p^{-}}(\Omega)$, the imbedding $W_{0}^{1, \vec{p}}(\Omega) \hookrightarrow L^{p^{-}}(\Omega)$ is compact.

Moreover, we consider the space

$$
\mathcal{T}_{0}^{1, \vec{p}}(\Omega)=\left\{u \text { measurable in } \Omega, T_{k}(u) \in W_{0}^{1, \vec{p}}(\Omega), \forall k>0\right\},
$$

where

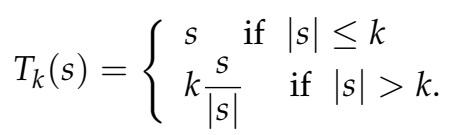

\section{Assumptions and Lemmas :}

In this section, we give the assumptions of our problem and some technical lemmas. Let $\Omega$ be a bounded open subset of $\mathbb{R}^{N}(N \geq 2)$ with Lipschitz continuous boundary $\partial \Omega$.

The functions $a_{i}: \Omega \times \mathbb{R} \times \mathbb{R}^{N} \rightarrow \mathbb{R}$ are Carathéodory functions satisfying the following conditions, for all $s \in \mathbb{R}, \xi \in \mathbb{R}^{N}, \xi^{\prime} \in \mathbb{R}^{N}$ and a. e. in $\Omega$,

$$
\begin{gathered}
\sum_{i=1}^{N} a_{i}(x, s, \xi) \xi_{i} \geq \alpha \sum_{i=1}^{N}\left|\xi_{i}\right|^{p_{i},} \\
\left|a_{i}(x, s, \xi)\right| \leq \beta\left[j_{i}(x)+|s|^{\frac{1}{p_{i}^{\prime}}}+\left|\xi_{i}\right|^{p_{i}-1}\right], \\
\left(a_{i}(x, s, \xi)-a_{i}\left(x, s, \xi^{\prime}\right)\right)\left(\xi_{i}-\xi_{i}^{\prime}\right)>0 \quad \text { for } \xi_{i} \neq \xi_{i}^{\prime},
\end{gathered}
$$


where $\alpha, \beta$ are some positive constants and $j_{i}$ is a positive function in $L^{p_{i}^{\prime}}(\Omega)$.

Moreover, we suppose that

$$
\phi_{i} \in C^{0}(\mathbb{R}, \mathbb{R}) \text { for } i=1, \ldots, N \text {. }
$$

and

$$
f \in L^{1}(\Omega)
$$

We consider the convex set

$$
K_{\psi}=\left\{u \in W_{0}^{1, \vec{p}}(\Omega), u \geq \psi \text { a.e. in } \Omega\right\}
$$

where $\psi$ is a measurable function with values in $\overline{\mathbb{R}}$ such that

$$
\psi^{+} \in W_{0}^{1, \vec{p}}(\Omega) \cap L^{\infty}(\Omega) .
$$

Lemma 3.1. ( [19]) Let $g \in L^{r}(\Omega)$ and $\operatorname{let}_{n} \in L^{r}(\Omega)$, $\left\|g_{n}\right\|_{L^{r}(\Omega)}<c, 1<r<+\infty$. If $g_{n}(x) \rightarrow g(x)$ a. e. in $\Omega$, then $g_{n} \rightarrow g$ weakly in $L^{r}(\Omega)$.

The following lemma generalizes lemma 5 in [13] to the anisotropic case. We utilize the method used in [2] and [13].

Lemma 3.2. : Assume that (3.1)-(3.3) hold and let $\left(u_{n}\right)_{n}$ be a sequence in $W_{0}^{1, \vec{p}}(\Omega)$ such that $u_{n} \rightarrow u$ in $W_{0}^{1, \vec{p}}(\Omega)$ and

$$
\lim _{n \rightarrow+\infty} \int_{\Omega}\left(a\left(x, u_{n}, \nabla u_{n}\right)-a\left(x, u_{n}, \nabla u\right)\right) \nabla\left(u_{n}-u\right) d x=0 .
$$

Then $u_{n} \rightarrow u$ strongly in $W_{0}^{1, \vec{p}}(\Omega)$ for a subsequence.

Proof Let $D_{n}=\left[a\left(x, u_{n}, \nabla u_{n}\right)-a\left(x, u_{n}, \nabla u\right)\right] \nabla\left(u_{n}-u\right)$, by (3.3), $D_{n}$ is a positive function and by (3.7), we have $D_{n} \rightarrow 0$ in $L^{1}(\Omega)$ as $n \rightarrow+\infty$. Since $u_{n} \rightarrow u$ in $W_{0}^{1, \vec{p}}(\Omega)$, using Remark 2.1, we have $u_{n} \rightarrow u$ strongly in $L^{p^{-}}(\Omega)$. Then $u_{n} \rightarrow u$ a. e. in $\Omega$ and $D_{n} \rightarrow 0$ a. e. in $\Omega$ for a subsequence. Thus there exists a subset $B$ of $\Omega$, of zero measure, such that for $x \in \Omega \backslash B, u(x)<+\infty,|\nabla u(x)|<+\infty,\left|j_{i}(x)\right|<+\infty, u_{n}(x) \rightarrow u(x)$ and $D_{n}(x) \rightarrow 0$. We have

$$
\begin{aligned}
D_{n}(x) & =\sum_{i=1}^{N}\left[a_{i}\left(x, u_{n}, \nabla u_{n}\right)-a_{i}\left(x, u_{n}, \nabla u\right)\right]\left[\partial_{i} u_{n}-\partial_{i} u\right] \\
& =\sum_{i=1}^{N}\left[a_{i}\left(x, u_{n}, \nabla u_{n}\right) \partial_{i} u_{n}+a_{i}\left(x, u_{n}, \nabla u\right) \partial_{i} u-a_{i}\left(x, u_{n}, \nabla u_{n}\right) \partial_{i} u-a_{i}\left(x, u_{n}, \nabla u\right) \partial_{i} u_{n}\right] \\
& \geq \alpha \sum_{i=1}^{N}\left|\partial_{i} u_{n}\right|^{p_{i}}+\alpha \sum_{i=1}^{N}\left|\partial_{i} u\right|^{p_{i}}-\beta \sum_{i=1}^{N}\left[j_{i}(x)+\left|u_{n}\right|^{\frac{1}{p_{i}^{\prime}}}+\left|\partial_{i} u_{n}\right|^{p_{i}-1}\right]\left|\partial_{i} u\right| \\
& -\beta \sum_{i=1}^{N}\left[j_{i}(x)+\left|u_{n}\right|^{\frac{1}{p_{i}^{\prime}}}+\left|\partial_{i} u\right|^{p_{i}-1}\right]\left|\partial_{i} u_{n}\right| . \\
\geq & \alpha \sum_{i=1}^{N}\left|\partial_{i} u_{n}\right|^{p_{i}}-c(x)\left[1+\sum_{i=1}^{N}\left|\partial_{i} u_{n}\right|^{p_{i}-1}+\sum_{i=1}^{N}\left|\partial_{i} u_{n}\right|\right] . \\
\geq & \sum_{\left\{i=1, \ldots, N: \partial_{i} u_{n} \neq 0\right\}}\left|\partial_{i} u_{n}\right|^{p_{i}}\left[\alpha-\frac{c(x)}{N\left|\partial_{i} u_{n}\right|^{p_{i}}}-\frac{c(x)}{N\left|\partial_{i} u_{n}\right|}-\frac{c(x)}{\left.N\left|\partial_{i} u_{n}\right|^{p_{i}-1}\right]}\right.
\end{aligned}
$$

where $c(x)$ is a function which doesn't depend on $n$.

Since $D_{n}(x) \rightarrow 0$ a. e. in $\Omega$, the last inequality implies that $\left(\partial_{i} u_{n}\right)_{n}$ is bounded uniformly with respect to $n$.

Letting $\xi_{i}^{*}$ be an accumulation point of $\left(\partial_{i} u_{n}\right)_{n}$ for $i=1, \ldots, N$, we have $\left|\xi_{i}^{*}\right|<+\infty$ and by the continuity of $a_{i}(x, . .$.$) , we obtain$

$$
\left(a_{i}\left(x, u, \xi^{*}\right)-a_{i}(x, u, \nabla u)\right)\left(\xi_{i}^{*}-\partial_{i} u\right)=0 .
$$


Using (3.3), we obtain $\xi_{i}^{*}=\partial_{i} u$ for $i=1, \ldots, N$. The uniqueness of the accumulation point implies that $\nabla u_{n} \rightarrow \nabla u$ a. e. in $\Omega$. Since the sequence $\left(a_{i}\left(x, u_{n}, \nabla u_{n}\right)\right)_{n}$ is bounded in $L^{p_{i}^{\prime}}(\Omega)$ and $a_{i}\left(x, u_{n}, \nabla u_{n}\right) \rightarrow a_{i}(x, u, \nabla u)$ a. e. in $\Omega$, by Lemma 3.1, we have $a_{i}\left(x, u_{n}, \nabla u_{n}\right)$ converges to $a_{i}(x, u, \nabla u)$ weakly in $L^{p_{i}^{\prime}}(\Omega)$ and a. e. in $\Omega$. As in [13], we have

$$
a_{i}\left(x, u_{n}(x), \nabla u_{n}(x)\right) \partial_{i} u_{n} \rightarrow a_{i}(x, u, \nabla u) \partial_{i} u \text { weakly in } L^{1}(\Omega) .
$$

For fixed $i=1, \ldots, N$, we set $y_{n}^{i}=\frac{1}{\alpha} a_{i}\left(x, u_{n}, \nabla u_{n}\right) \partial_{i} u_{n}$ and $y^{i}=\frac{1}{\alpha} a_{i}(x, u, \nabla u) \partial_{i} u$, using Fatou's lemma, we get

$$
\int_{\Omega} 2 y^{i} d x \leq \liminf _{n \rightarrow+\infty} \int_{\Omega}\left(y_{n}^{i}+y^{i}-\frac{1}{2^{p_{i}-1}}\left|\partial_{i} u_{n}-\partial_{i} u\right|^{p_{i}}\right) d x .
$$

Then, we have $0 \leq-\limsup _{n \rightarrow+\infty} \int_{\Omega}\left|\partial_{i} u_{n}-\partial_{i} u\right|^{p_{i}} d x$. We deduce $\int_{\Omega}\left|\partial_{i} u_{n}-\partial_{i} u\right|^{p_{i}} d x \rightarrow 0$ as $n \rightarrow+\infty$.

Consequently, we conclude that $u_{n} \rightarrow u$ in $W_{0}^{1, \vec{p}}(\Omega)$, the proof is complete.

Lemma 3.3. If $u \in W_{0}^{1, \vec{p}}(\Omega)$, then $\sum_{i=1}^{N} \int_{\Omega} \partial_{i} u d x=0$.

Proof: Since $u \in W_{0}^{1, \vec{p}}(\Omega)$, there exists $u_{k} \in C_{0}^{\infty}(\Omega)$ such that $u_{k} \rightarrow u$ strongly in $W_{0}^{1, \vec{p}}(\Omega)$. Moreover, since $u_{k} \in C_{0}^{\infty}(\Omega)$, by Green's Formula, we have

$$
\sum_{i=1}^{N} \int_{\Omega} \partial_{i} u_{k} d x=\int_{\partial \Omega} u_{k} \cdot \vec{n} d s=0
$$

Since $\partial_{i} u_{k} \rightarrow \partial_{i} u$ strongly in $L^{p_{i}}(\Omega)$, we have $\partial_{i} u_{k} \rightarrow \partial_{i} u$ strongly in $L^{1}(\Omega)$.

We pass to limit in (3.8), we conclude that $\sum_{i=1}^{N} \int_{\Omega} \partial_{i} u d x=0$.

\section{Main result}

Definition 4.1. A function $u \in \mathcal{T}_{0}^{1, \vec{p}}(\Omega)$ such that $u \geq \psi$ a. e. in $\Omega$ is an entropy solution of the problem (1.1) if

$$
\sum_{i=1}^{N} \int_{\Omega}\left[a_{i}(x, u, \nabla u) \partial_{i} T_{k}(u-\varphi) d x+\phi_{i}(u) \partial_{i} T_{k}(u-\varphi) d x\right] \leq \int_{\Omega} f T_{k}(u-\varphi) d x
$$

for all $\varphi \in K_{\psi}(\Omega) \cap L^{\infty}(\Omega)$.

Theorem 4.1. Assume that (3.1)-(3.6) hold. Then there exists at least an entropy solution of problem (1.1).

Proof:

Step1. Approximate problems. We consider the following approximate problems

$$
\left\{\begin{array}{l}
u_{n} \in K_{\psi} . \\
\sum_{i=1}^{N} \int_{\Omega} a_{i}\left(x, u_{n}, \nabla u_{n}\right) \partial_{i}\left(u_{n}-v\right) d x+\sum_{i=1}^{N} \int_{\Omega} \phi_{i}^{n}\left(u_{n}\right) \partial_{i}\left(u_{n}-v\right) d x \leq \int_{\Omega} f_{n}\left(u_{n}-v\right) d x, \\
\forall v \in K_{\psi} \text { and } \forall k>0,
\end{array}\right.
$$

where $f_{n}=T_{n}(f)$ and $\phi_{i}^{n}(s)=\phi_{i}\left(T_{n}(s)\right)$.

Lemma 4.1. We consider the operator $\Phi_{n}: K_{\psi} \rightarrow W^{-1, \overrightarrow{p^{\prime}}}(\Omega)$ defined by

$<\Phi_{n} u, v>=\sum_{i=1}^{N} \int_{\Omega} \phi_{i}\left(T_{n}(u)\right) \partial_{i} v d x \quad$ for all $u \in K_{\psi}$ and $v \in W_{0}^{1, \vec{p}}(\Omega)$. 
The operator $B_{n}=A+\Phi_{n}$ is pseudo-monotone and coercive in the following sense; there exists $v_{0} \in K_{\psi}$ such that $\frac{<B_{n} v, v-v_{0}>}{\|v\|_{W_{0}^{1,}, \vec{p}}(\Omega)} \rightarrow+\infty$ if $\|v\|_{W_{0}^{1, \vec{p}}(\Omega)} \rightarrow+\infty$ and $v \in K_{\psi}$.

For the proof of Lemma 4.1, (see Appendix).

Proposition 4.1. Under the conditions (3.1)-(3.6), there exists at least one solution of the problem (4.1).

Proof. Thanks to Lemma 4.1 and Theorem 8.2 chapiter 2 in [19], there exists at least one solution to the problem (4.1).

\section{Step2. A priori estimate.}

Proposition 4.2. Assume that (3.1)- (3.6) hold and if $u_{n}$ is a solution of the approximate problem (4.1). Then there exists a constant $C$ such that

$$
\sum_{i=1}^{N} \int_{\Omega}\left|\partial_{i} T_{k}\left(u_{n}\right)\right|^{p_{i}} d x \leq C(k+1) \quad \forall k>0
$$

Proof. Let $v=u_{n}-\eta T_{k}\left(u_{n}^{+}-\psi^{+}\right)$where $\eta \geq 0$. Since $v \in W_{0}^{1, \vec{p}}(\Omega)$ and for all $\eta$ small enough, we have $v \in K_{\psi}$. We take $v$ as test function in problem (4.1), we have

$\sum_{i=1}^{N} \int_{\Omega} a_{i}\left(x, u_{n}, \nabla u_{n}\right) \partial_{i} T_{k}\left(u_{n}^{+}-\psi^{+}\right) d x+\sum_{i=1}^{N} \int_{\Omega} \phi_{i}^{n}\left(u_{n}\right) \partial_{i} T_{k}\left(u_{n}^{+}-\psi^{+}\right) d x \leq \int_{\Omega} f_{n} T_{k}\left(u_{n}^{+}-\psi^{+}\right) d x$.

Which implies that

$$
\sum_{i=1}^{N} \int_{\Omega} a_{i}\left(x, u_{n}, \nabla u_{n}\right) \partial_{i} T_{k}\left(u_{n}^{+}-\psi^{+}\right) d x \leq \int_{\Omega} f_{n} T_{k}\left(u_{n}^{+}-\psi^{+}\right) d x+\sum_{i=1}^{N} \int_{\Omega}\left|\phi_{i}^{n}\left(u_{n}\right)\right|\left|\partial_{i} T_{k}\left(u_{n}^{+}-\psi^{+}\right)\right| d x .
$$

Since $\partial_{i} T_{k}\left(u_{n}^{+}-\psi^{+}\right)=0$ on the set $\left\{u_{n}^{+}-\psi^{+}>k\right\}$, we have

$$
\begin{aligned}
\sum_{i=1}^{N} \int_{\left\{u_{n}^{+}-\psi^{+} \leq k\right\}} a_{i}\left(x, u_{n}, \nabla u_{n}\right) \partial_{i}\left(u_{n}^{+}-\psi^{+}\right) d x \leq \int_{\Omega} & f_{n} T_{k}\left(u_{n}^{+}-\psi^{+}\right) d x \\
& +\sum_{i=1}^{N} \int_{\left\{u_{n}^{+}-\psi^{+} \leq k\right\}}\left|\phi_{i}^{n}\left(u_{n}\right)\right|\left|\partial_{i}\left(u_{n}^{+}-\psi^{+}\right)\right| d x,
\end{aligned}
$$

thus, we can write

$$
\begin{aligned}
\sum_{i=1}^{N} \int_{\left\{u_{n}^{+}-\psi^{+} \leq k\right\}} a_{i}\left(x, u_{n}^{+}\right. & \left., \nabla u_{n}^{+}\right) \partial_{i} u_{n}^{+} d x \leq \int_{\Omega} f_{n} T_{k}\left(u_{n}^{+}-\psi^{+}\right) d x+\sum_{i=1}^{N} \int_{\left\{u_{n}^{+}-\psi^{+} \leq k\right\}}\left|\phi_{i}^{n}\left(u_{n}\right)\right|\left|\partial_{i} u_{n}^{+}\right| d x \\
& +\sum_{i=1}^{N} \int_{\left\{u_{n}^{+}-\psi^{+} \leq k\right\}}\left|\phi_{i}^{n}\left(u_{n}\right)\right|\left|\partial_{i} \psi^{+}\right| d x+\sum_{i=1}^{N} \int_{\left\{u_{n}^{+}-\psi^{+} \leq k\right\}} a_{i}\left(x, u_{n}^{+}, \nabla u_{n}^{+}\right) \partial_{i} \psi^{+} d x
\end{aligned}
$$

Thanks to Young's inequalities, we obtain

$$
\begin{aligned}
\sum_{i=1}^{N} \int_{\left\{u_{n}^{+}-\psi^{+} \leq k\right\}} & a_{i}\left(x, u_{n}^{+}, \nabla u_{n}^{+}\right) \partial_{i} u_{n}^{+} d x \leq \int_{\Omega} f_{n} T_{k}\left(u_{n}^{+}-\psi^{+}\right) d x \\
& +C_{1}(\alpha) \sum_{i=1}^{N} \int_{\left\{u_{n}^{+}-\psi^{+} \leq k\right\}}\left|\phi_{i}^{n}\left(T_{k+\|\psi\|_{\infty}}\left(u_{n}\right)\right)\right|^{p_{i}^{\prime}} d x+\frac{\alpha}{6} \sum_{i=1}^{N} \int_{\left\{u_{n}^{+}-\psi^{+} \leq k\right\}}\left|\partial_{i} u_{n}^{+}\right|^{p_{i}} d x \\
& +\sum_{i=1}^{N} \int_{\left\{u_{n}^{+}-\psi^{+} \leq k\right\}}\left|\phi_{i}^{n}\left(T_{k+\|\psi\|_{\infty}}\left(u_{n}\right)\right)\right|\left|\partial_{i} \psi^{+}\right| d x \\
& +\sum_{i=1}^{N} \int_{\left\{u_{n}^{+}-\psi^{+} \leq k\right\}} \beta\left[j_{i}(x)+\left|u_{n}^{+}\right|^{\frac{1}{p_{i}^{\prime}}}+\left|\partial_{i} u_{n}^{+}\right|^{p_{i}-1}\right]\left|\partial_{i} \psi^{+}\right| d x
\end{aligned}
$$

Thanks to (3.2), we have

$$
\sum_{i=1}^{N} \int_{\left\{u_{n}^{+}-\psi^{+} \leq k\right\}} a_{i}\left(x, u_{n}, \nabla u_{n}\right) \partial_{i} u_{n}^{+} d x \leq \int_{\Omega} f_{n} T_{k}\left(u_{n}^{+}-\psi^{+}\right) d x
$$




$$
\begin{aligned}
& +C_{2}(\alpha) \sum_{i=1}^{N} \int_{\left\{u_{n}^{+}-\psi^{+} \leq k\right\}}\left|\phi_{i}^{n}\left(T_{k+\|\psi\|_{\infty}}\left(u_{n}\right)\right)\right|^{p_{i}^{\prime}} d x+\frac{\alpha}{6} \sum_{i=1}^{N} \int_{\left\{u_{n}^{+}-\psi^{+} \leq k\right\}}\left|\partial_{i} u_{n}^{+}\right|^{p_{i}} d x \\
& +\sum_{i=1}^{N} \int_{\left\{u_{n}^{+}-\psi^{+} \leq k\right\}}\left|\phi_{i}^{n}\left(T_{k+\|\psi\|_{\infty}}\left(u_{n}\right)\right)\right|\left|\partial_{i} \psi^{+}\right| d x+\beta \sum_{i=1}^{N} \int_{\left\{u_{n}^{+}-\psi^{+} \leq k\right\}} j_{i}(x)\left|\partial_{i} \psi^{+}\right| d x \\
& +\beta \sum_{i=1}^{N} \int_{\left\{u_{n}^{+}-\psi^{+} \leq k\right\}}\left|u_{n}^{+}\right|^{\frac{1}{p_{i}^{\prime}}}\left|\partial_{i} \psi^{+}\right| d x+\beta \sum_{i=1}^{N} \int_{\left\{u_{n}^{+}-\psi^{+} \leq k\right\}}\left|\partial_{i} u_{n}^{+}\right|^{p_{i}-1}\left|\partial_{i} \psi^{+}\right| d x
\end{aligned}
$$

Using young's inequality, we get

$$
\begin{aligned}
\sum_{i=1}^{N} \int_{\left\{u_{n}^{+}-\psi^{+} \leq k\right\}} & a_{i}\left(x, u_{n}, \nabla u_{n}\right) \partial_{i} u_{n}^{+} d x \leq \int_{\Omega} f_{n} T_{k}\left(u_{n}^{+}-\psi^{+}\right) d x \\
& +\left.C_{3}(\alpha) \sum_{i=1}^{N} \int_{\left\{u_{n}^{+}-\psi^{+} \leq k\right\}}\left|\phi_{i}^{n}\left(T_{k+\|\psi\|_{\infty}}\left(u_{n}\right)\right)\right|\right|^{\prime} d x+\frac{\alpha}{6} \sum_{i=1}^{N} \int_{\left\{u_{n}^{+}-\psi^{+} \leq k\right\}}\left|\partial_{i} u_{n}^{+}\right|^{p_{i}} d x \\
& +\sum_{i=1}^{N} \int_{\left\{u_{n}^{+}-\psi^{+} \leq k\right\}}\left|\phi_{i}^{n}\left(T_{k+\|\psi\|_{\infty}}\left(u_{n}\right)\right)\right|\left|\partial_{i} \psi^{+}\right| d x+\beta \sum_{i=1}^{N} \int_{\left\{u_{n}^{+}-\psi^{+} \leq k\right\}}\left|j_{i}(x)\right|\left|\partial_{i} \psi^{+}\right| d x \\
& +\beta C_{4} \sum_{i=1}^{N} \int_{\left\{u_{n}^{+}-\psi^{+} \leq k\right\}}\left|u_{n}^{+}\right| d x+\beta \frac{\alpha}{6 \beta} \sum_{i=1}^{N} \int_{\left\{u_{n}^{+}-\psi^{+} \leq k\right\}}\left|\partial_{i} \psi^{+}\right| d x \\
& +\beta \frac{\alpha}{6 \beta} \sum_{i=1}^{N} \int_{\left\{u_{n}^{+}-\psi^{+} \leq k\right\}}\left|\partial_{i} u_{n}^{+}\right|^{p_{i}} d x+C_{5}(\alpha) \sum_{i=1}^{N} \int_{\left\{u_{n}^{+}-\psi^{+} \leq k\right\}}\left|\partial_{i} \psi^{+}\right|^{p_{i}} d x
\end{aligned}
$$

Using (3.1), (3.4), (3.5) and (3.6), we get

$$
\sum_{i=1}^{N} \int_{\left\{u_{n}^{+}-\psi^{+} \leq k\right\}}\left|\partial_{i} u_{n}^{+}\right|^{p_{i}} d x \leq C k+C^{\prime}
$$

Since $\left\{x \in \Omega, u^{+} \leq k\right\} \subset\left\{x \in \Omega, u^{+}-\psi^{+} \leq k+\left\|\psi^{+}\right\|_{\infty}\right\}$, then

$$
\sum_{i=1}^{N} \int_{\Omega}\left|\partial_{i} T_{k}\left(u_{n}^{+}\right)\right|^{p_{i}} d x=\sum_{i=1}^{N} \int_{\left\{u^{+} \leq k\right\}}\left|\partial_{i} u_{n}^{+}\right|^{p_{i}} d x \leq \sum_{i=1}^{N} \int_{\left\{u^{+}-\psi^{+} \leq k+\left\|\psi^{+}\right\|_{\infty}\right\}}\left|\partial_{i} u_{n}^{+}\right|^{p_{i}} d x .
$$

Thus, by (4.2), we obtain

$$
\sum_{i=1}^{N} \int_{\Omega}\left|\partial_{i} T_{k}\left(u_{n}^{+}\right)\right|^{p_{i}} d x \leq\left(k+\left\|\psi^{+}\right\|_{\infty}\right) C+C^{\prime} \quad \forall k>0 .
$$

Similarly, taking $v=u_{n}+T_{k}\left(u_{n}^{-}\right)$as test function in approximate problem (4.1), we obtain

$$
\sum_{i=1}^{N} \int_{\Omega}\left|\partial_{i} T_{k}\left(u_{n}^{-}\right)\right|^{p_{i}} d x \leq C^{\prime \prime}(k+1)
$$

Combining (4.3) and (4.4), we get

$$
\sum_{i=1}^{N} \int_{\Omega}\left|\partial T_{k}\left(u_{n}\right)\right|^{p_{i}} d x \leq\left(k+\left\|\psi^{+}\right\|_{\infty}+1\right) C^{\prime} \quad \forall k>0
$$

\section{Step3. Strong convergence of truncations.}

Proposition 4.3. If $u_{n}$ is a solution of approximate problem (4.1). Then there exists a measurable function $u$ and a subsequence of $u_{n}$ such that

$$
T_{k}\left(u_{n}\right) \rightarrow T_{k}(u) \text { strongly in } W_{0}^{1, \vec{p}}(\Omega) .
$$

Proof. Using Proposition 4.1, we obtain

$$
\left\|T_{k}\left(u_{n}\right)\right\|_{W_{0}^{1, \vec{p}}(\Omega)} \leq C\left(k+\left\|\psi^{+}\right\|_{\infty}+1\right)^{\frac{1}{p_{-}}} .
$$


Now, we will prove that $\left(u_{n}\right)_{n}$ is a Cauchy sequence in measure in $\Omega$. For all $\lambda>0$, we have $\left\{\left|u_{n}-u_{m}\right|>\lambda\right\} \subset\left\{\left|u_{n}\right|>k\right\} \cup\left\{\left|u_{m}\right|>k\right\} \cup\left\{\left|T_{k}\left(u_{n}\right)-T_{k}\left(u_{m}\right)\right|>\lambda\right\}$

which implies that

$$
\begin{gathered}
\operatorname{meas}\left\{\left|u_{n}-u_{m}\right|>\lambda\right\} \leq \operatorname{meas}\left\{\left|u_{n}\right|>k\right\}+\text { meas }\left\{\left|u_{m}\right|>k\right\} \\
+ \text { meas }\left\{\left|T_{k}\left(u_{n}\right)-T_{k}\left(u_{m}\right)\right|>\lambda\right\} .
\end{gathered}
$$

By Hölder's inequality, Remark 2.1 and (4.5), we have

$$
\begin{aligned}
\text { k.meas }\left\{\left|u_{n}\right|>k\right\}=\int_{\left\{\left|u_{n}\right|>k\right\}}\left|T_{k}\left(u_{n}\right)\right| d x & \leq \int_{\Omega}\left|T_{k}\left(u_{n}\right)\right| d x \\
& \leq(\operatorname{meas}(\Omega))^{\frac{1}{p^{-1}}}\left\|T_{k}\left(u_{n}\right)\right\|_{L^{p^{-}}(\Omega)} \\
& \leq C(\operatorname{meas}(\Omega))^{\frac{1}{p^{-}}}\left\|T_{k}\left(u_{n}\right)\right\|_{W_{0}^{1, \vec{p}}(\Omega)} \\
& \leq C\left(k+\left\|\psi^{+}\right\|_{\infty}+1\right)^{\frac{1}{p^{-}}} .
\end{aligned}
$$

Then meas $\left\{\left|u_{n}\right|>k\right\} \leq C\left(\frac{1}{k^{-1+p^{-}}}+\frac{1+\left\|\psi^{+}\right\|_{\infty}}{k^{p^{-}}}\right)^{\frac{1}{p^{-}}} \rightarrow 0$ as $k \rightarrow+\infty$. Which implies that, for all $\varepsilon>0$, there exists $k_{0}$ such that $\forall k>k_{0}$, we have

$$
\text { meas }\left\{\left|u_{n}\right|>k\right\} \leq \frac{\varepsilon}{3} \text { and meas }\left\{\left|u_{m}\right|>k\right\} \leq \frac{\varepsilon}{3} .
$$

Moreover, since the sequence $\left(T_{k}\left(u_{n}\right)\right)_{n}$ is bounded in $W_{0}^{1, \vec{p}}(\Omega)$, there exists a subsequence $\left(T_{k}\left(u_{n}\right)\right)_{n}$ such that $T_{k}\left(u_{n}\right)$ converges to $v_{k}$ a.e. in $\Omega$, weakly in $W_{0}^{1, \vec{p}}(\Omega)$ and strongly in $L^{p^{-}}(\Omega)$ as $n$ tends to $+\infty$. Then the sequence $\left(T_{k}\left(u_{n}\right)\right)_{n}$ is a Cauchy sequence in measure in $\Omega$, thus $\forall \lambda>0$, there exists $n_{0}$ such that

$$
\text { meas }\left\{\left|T_{k}\left(u_{n}\right)-T_{k}\left(u_{m}\right)\right|>\lambda\right\} \leq \frac{\varepsilon}{3}, \forall n, m \geq n_{0} .
$$

Combining (4.6), (4.7) and (4.8), then for all $\lambda>0$ and for all $\varepsilon>0$, we have

$$
\text { meas }\left\{\left|u_{n}-u_{m}\right|>\lambda\right\} \leq \varepsilon \quad \forall n, m \geq n_{0} .
$$

Then $\left(u_{n}\right)_{n}$ is a Cauchy sequence in measure in $\Omega$, then there exists a subsequence denoted by $\left(u_{n}\right)_{n}$ such that $u_{n}$ converges to a measurable function $u$ a.e. in $\Omega$ and

$$
T_{k}\left(u_{n}\right) \rightarrow T_{k}(u) \text { weakly in } W_{0}^{1, \vec{p}}(\Omega) \text { and a.e. in } \Omega \quad \forall k>0 .
$$

It remains to prove that

$$
\lim _{n \rightarrow \infty} \sum_{i=1}^{N} \int_{\Omega}\left(a_{i}\left(x, T_{k}\left(u_{n}\right), \nabla T_{k}\left(u_{n}\right)\right)-a_{i}\left(x, T_{k}\left(u_{n}\right), \nabla T_{k}(u)\right)\right)\left(\partial_{i} T_{k}\left(u_{n}\right)-\partial_{i} T_{k}(u)\right) d x=0 .
$$

Let us take $v=u_{n}+T_{1}\left(u_{n}-T_{m}\left(u_{n}\right)\right)^{-}$as test function in approximate problem (4.1), we obtain

$$
\begin{gathered}
-\sum_{i=1}^{N} \int_{\Omega} a_{i}\left(x, u_{n}, \nabla u_{n}\right) \partial_{i} T_{1}\left(u_{n}-T_{m}\left(u_{n}\right)\right)^{-} d x-\sum_{i=1}^{N} \int_{\Omega} \phi_{i}^{n}\left(u_{n}\right) \partial_{i} T_{1}\left(u_{n}-T_{m}\left(u_{n}\right)\right)^{-} d x \\
\leq-\int_{\Omega} f_{n} T_{1}\left(u_{n}-T_{m}\left(u_{n}\right)\right)^{-} d x .
\end{gathered}
$$

$$
\begin{aligned}
& \text { Then } \\
& \sum_{i=1}^{N} \int_{\left\{-(m+1) \leq u_{n} \leq-m\right\}} a_{i}\left(x, u_{n}, \nabla u_{n}\right) \partial_{i} u_{n} d x+\sum_{i=1}^{N} \int_{\left\{-(m+1) \leq u_{n} \leq-m\right\}} \phi_{i}\left(u_{n}\right) \partial_{i} u_{n} d x \\
& \leq-\int_{\Omega} f_{n} T_{1}\left(u_{n}-T_{m}\left(u_{n}\right)\right)^{-} d x .
\end{aligned}
$$


We set $\Phi_{i}^{n}(s)=\int_{0}^{s} \phi_{i}^{n}(t) \chi_{\{-(m+1) \leq t \leq-m\}} d t$. Then by Green's formula, we have

$\sum_{i=1}^{N} \int_{\left\{-(m+1) \leq u_{n} \leq-m\right\}} \phi_{i}\left(u_{n}\right) \partial_{i} u_{n} d x=\sum_{i=1}^{N} \int_{\Omega} \partial_{i} \Phi_{i}^{n}\left(u_{n}\right) d x=0$.

Then, we get

$\sum_{i=1}^{N} \int_{\left\{-(m+1) \leq u_{n} \leq-m\right\}} a_{i}\left(x, u_{n}, \nabla u_{n}\right) \partial_{i} u_{n} d x \leq-\int_{\Omega} f_{n} T_{1}\left(u_{n}-T_{m}\left(u_{n}\right)\right)^{-} d x$

By Lebesgue's theorem, we have

$$
\lim _{m \rightarrow+\infty} \limsup _{n \rightarrow+\infty} \int_{\Omega} f_{n} T_{1}\left(u_{n}-T_{m}\left(u_{n}\right)\right)^{-} d x=0
$$

Then, we have

$$
\lim _{m \rightarrow+\infty} \limsup _{n \rightarrow+\infty} \sum_{i=1}^{N} \int_{\left\{-(m+1) \leq u_{n} \leq-m\right\}} a_{i}\left(x, u_{n}, \nabla u_{n}\right) \partial_{i} u_{n} d x=0 .
$$

Similarly, taking $v=u_{n}-\eta T_{1}\left(u_{n}-T_{m}\left(u_{n}\right)\right)^{+}$as test function in approximate problem (4.1), we get

$$
\lim _{m \rightarrow \infty} \limsup _{n \rightarrow \infty} \sum_{i=1}^{N} \int_{\left\{m \leq u_{n} \leq m+1\right\}} a_{i}\left(x, u_{n}, \nabla u_{n}\right) \partial_{i} u_{n} d x=0 .
$$

We consider the following function of one real variable:

$$
h_{m}(s)=\left\{\begin{array}{l}
1 \quad \text { if }|s| \leq m \\
0 \quad \text { if }|s| \geq m+1 \\
m+1-|s| \text { if } m \leq|s| \leq m+1
\end{array}\right.
$$

where $m>k$.

Let $\varphi=u_{n}-\eta\left(T_{k}\left(u_{n}\right)-T_{k}(u)\right)^{+} h_{m}\left(u_{n}\right)$ as test function in approximate problem (4.1), we obtain

$$
\begin{gathered}
\sum_{i=1}^{N} \int_{\Omega} a_{i}\left(x, u_{n}, \nabla u_{n}\right) \partial_{i}\left(T_{k}\left(u_{n}\right)-T_{k}(u)\right)^{+} h_{m}\left(u_{n}\right) d x \\
+\sum_{i=1}^{N} \int_{\Omega} a_{i}\left(x, u_{n}, \nabla u_{n}\right)\left(T_{k}\left(u_{n}\right)-T_{k}(u)\right)^{+} \partial_{i} u_{n} h_{m}^{\prime}\left(u_{n}\right) d x \\
+\sum_{i=1}^{N} \int_{\Omega} \phi_{i}^{n}\left(u_{n}\right) \partial_{i}\left(T_{k}\left(u_{n}\right)-T_{k}(u)\right)^{+} h_{m}\left(u_{n}\right) d x+\sum_{i=1}^{N} \int_{\Omega} \phi_{i}^{n}\left(u_{n}\right) \partial_{i} u_{n}\left(T_{k}\left(u_{n}\right)-T_{k}(u)\right)^{+} h_{m}^{\prime}\left(u_{n}\right) d x \\
\leq \int_{\Omega} f_{n}\left(T_{k}\left(u_{n}\right)-T_{k}(u)\right)^{+} h_{m}\left(u_{n}\right) d x
\end{gathered}
$$

By (4.12) and (4.13), we have the second integral in (4.14) converges to zero as $n$ and $m$ tend to $+\infty$. Since $h_{m}\left(u_{n}\right)=0$ if $\left|u_{n}\right|>m+1$, we have

$$
\sum_{i=1}^{N} \int_{\Omega} \phi_{i}^{n}\left(u_{n}\right) \partial_{i}\left(T_{k}\left(u_{n}\right)-T_{k}(u)\right)^{+} h_{m}\left(u_{n}\right) d x=\sum_{i=1}^{N} \int_{\Omega} \phi_{i}\left(T_{m+1}\left(u_{n}\right)\right) h_{m}\left(u_{n}\right) \partial_{i}\left(T_{k}\left(u_{n}\right)-T_{k}(u)\right)^{+} d x .
$$

Using Lebesgue's theorem, we have $\phi_{i}^{n}\left(T_{m+1}\left(u_{n}\right)\right) h_{m}\left(u_{n}\right) \rightarrow \phi_{i}\left(T_{m+1}(u)\right) h_{m}(u)$ in $L^{p_{i}^{\prime}}(\Omega)$ and $\partial_{i} T_{k}\left(u_{n}\right) \rightarrow \partial_{i} T_{k}(u)$ weakly in $L^{p_{i}}(\Omega)$ as $n$ tends to $+\infty$, then the third integral in (4.14) converges to zero as $n$ and $m$ tend to $+\infty$. Using (3.1), (4.12), (4.13) and Lebesgue's theorem, we obtain

$$
\lim _{m \rightarrow+\infty} \lim _{n \rightarrow+\infty} \sum_{i=1}^{N} \int_{\left\{-(m+1) \leq u_{n} \leq-m\right\}}\left|\partial_{i} u_{n}\right|^{p_{i}}\left(T_{k}\left(u_{n}\right)-T_{k}(u)\right)^{+} d x=0
$$


and

$$
\lim _{m \rightarrow+\infty} \lim _{n \rightarrow+\infty} \sum_{i=1}^{N} \int_{\left\{m \leq u_{n} \leq m+1\right\}}\left|\partial_{i} u_{n}\right|^{p_{i}}\left(T_{k}\left(u_{n}\right)-T_{k}(u)\right)^{+} d x=0
$$

We deduce that

$$
\lim _{m \rightarrow+\infty} \lim _{n \rightarrow+\infty} \sum_{i=1}^{N} \int_{\Omega} a_{i}\left(x, u_{n}, \nabla u_{n}\right) \partial_{i}\left(T_{k}\left(u_{n}\right)-T_{k}(u)\right)^{+} h_{m}\left(u_{n}\right) d x \leq 0,
$$

which implies that

$$
\begin{gathered}
\lim _{m \rightarrow+\infty} \lim _{n \rightarrow+\infty} \sum_{i=1}^{N} \int_{\left\{T_{k}\left(u_{n}\right)-T_{k}(u) \geq 0,\left|u_{n}\right| \leq k\right\}} a_{i}\left(x, u_{n}, \nabla u_{n}\right) \partial_{i}\left(T_{k}\left(u_{n}\right)-T_{k}(u)\right) h_{m}\left(u_{n}\right) d x \\
-\lim _{m \rightarrow+\infty} \lim _{n \rightarrow+\infty} \sum_{i=1}^{N} \int_{\left\{T_{k}\left(u_{n}\right)-T_{k}(u) \geq 0,\left|u_{n}\right|>k\right\}} a_{i}\left(x, u_{n}, \nabla u_{n}\right) \partial_{i} T_{k}(u) h_{m}\left(u_{n}\right) d x \leq 0 .
\end{gathered}
$$

Since $h_{m}\left(u_{n}\right)=0$ in $\left\{\left|u_{n}\right|>m+1\right\}$, we have

$$
\begin{gathered}
\sum_{i=1}^{N} \int_{\left\{T_{k}\left(u_{n}\right)-T_{k}(u) \geq 0,\left|u_{n}\right|>k\right\}} a_{i}\left(x, u_{n}, \nabla u_{n}\right) \partial_{i} T_{k}(u) h_{m}\left(u_{n}\right) d x \\
=\sum_{i=1}^{N} \int_{\left\{T_{k}\left(u_{n}\right)-T_{k}(u) \geq 0,\left|u_{n}\right|>k\right\}} a_{i}\left(x, T_{m+1}\left(u_{n}\right), \nabla T_{m+1}\left(u_{n}\right)\right) \partial_{i} T_{k}(u) h_{m}\left(u_{n}\right) d x .
\end{gathered}
$$

Since $\left(a_{i}\left(x, T_{m+1}\left(u_{n}\right), \nabla T_{m+1}\left(u_{n}\right)\right)\right)_{n \geq 0}$ is bounded in $L^{p_{i}^{\prime}}(\Omega)$, we have $a_{i}\left(x, T_{m+1}\left(u_{n}\right), \nabla T_{m+1}\left(u_{n}\right)\right)$ converges to $X_{m}^{i}$ weakly in $L^{p_{i}^{\prime}}(\Omega)$. Then

$$
\begin{array}{r}
\lim _{m \rightarrow+\infty} \lim _{n \rightarrow+\infty} \sum_{i=1}^{N} \int_{\left\{T_{k}\left(u_{n}\right)-T_{k}(u) \geq 0,\left|u_{n}\right|>k\right\}} a_{i}\left(x, T_{m+1}\left(u_{n}\right), \nabla T_{m+1}\left(u_{n}\right)\right) \partial_{i} T_{k}(u) h_{m}\left(u_{n}\right) d x \\
=\lim _{m \rightarrow+\infty} \sum_{i=1}^{N} \int_{\{|u|>k\}} X_{m}^{i} \partial_{i} T_{k}(u) h_{m}(u) d x=0,
\end{array}
$$

which implies

$$
\lim _{m \rightarrow+\infty} \lim _{n \rightarrow+\infty} \sum_{i=1}^{N} \int_{\left\{T_{k}\left(u_{n}\right)-T_{k}(u) \geq 0\right\}} a_{i}\left(x, T_{k}\left(u_{n}\right), \nabla T_{k}\left(u_{n}\right)\right) \partial_{i}\left(T_{k}\left(u_{n}\right)-T_{k}(u)\right) h_{m}\left(u_{n}\right) d x \leq 0 .
$$

Moreover, we have $a_{i}\left(x, T_{k}\left(u_{n}\right), \nabla T_{k}(u)\right) h_{m}\left(u_{n}\right) \rightarrow a_{i}\left(x, T_{k}(u), \nabla T_{k}(u)\right) h_{m}(u)$ in $L^{p_{i}^{\prime}}(\Omega)$ and $\partial_{i}\left(T_{k}\left(u_{n}\right)-T_{k}(u)\right) \rightarrow 0$ weakly in $L^{p_{i}}(\Omega)$, then

$$
\lim _{m \rightarrow+\infty} \lim _{n \rightarrow+\infty} \sum_{i=1}^{N} \int_{\left\{T_{k}\left(u_{n}\right)-T_{k}(u) \geq 0\right\}} a_{i}\left(x, T_{k}\left(u_{n}\right), \nabla T_{k}(u)\right) \partial_{i}\left(T_{k}\left(u_{n}\right)-T_{k}(u)\right) h_{m}\left(u_{n}\right) d x=0 .
$$

Combining (3.3), (4.17) and (4.18), we deduce

$$
\begin{gathered}
\lim _{m \rightarrow+\infty} \lim _{n \rightarrow+\infty} \sum_{i=1}^{N} \int_{\left\{T_{k}\left(u_{n}\right)-T_{k}(u) \geq 0\right\}}\left(a_{i}\left(x, T_{k}\left(u_{n}\right), \nabla T_{k}\left(u_{n}\right)\right)-a_{i}\left(x, T_{k}\left(u_{n}\right), \nabla T_{k}(u)\right)\right) \\
\partial_{i}\left(T_{k}\left(u_{n}\right)-T_{k}(u)\right) h_{m}\left(u_{n}\right) d x=0 .
\end{gathered}
$$

Similarly, we take $\varphi=u_{n}+\left(T_{k}\left(u_{n}\right)-T_{k}(u)\right)^{-} h_{m}\left(u_{n}\right)$ as test function in approximate problem (4.1), we obtain,

$$
\begin{gathered}
\lim _{m \rightarrow+\infty} \lim _{n \rightarrow+\infty} \sum_{i=1}^{N} \int_{\left\{T_{k}\left(u_{n}\right)-T_{k}(u) \leq 0\right\}}\left(a_{i}\left(x, T_{k}\left(u_{n}\right), \nabla T_{k}\left(u_{n}\right)\right)-a_{i}\left(x, T_{k}\left(u_{n}\right), \nabla T_{k}(u)\right)\right) \\
\partial_{i}\left(T_{k}\left(u_{n}\right)-T_{k}(u)\right) h_{m}\left(u_{n}\right) d x=0 .
\end{gathered}
$$


Combining (4.19) and (4.20) we get

$$
\begin{array}{r}
\lim _{m \rightarrow+\infty} \lim _{n \rightarrow+\infty} \sum_{i=1}^{N} \int_{\Omega}\left(a_{i}\left(x, T_{k}\left(u_{n}\right), \nabla T_{k}\left(u_{n}\right)\right)-a_{i}\left(x, T_{k}\left(u_{n}\right), \nabla T_{k}(u)\right)\right) \\
\partial_{i}\left(T_{k}\left(u_{n}\right)-T_{k}(u)\right) h_{m}\left(u_{n}\right) d x=0 .
\end{array}
$$

Now, we prove $\lim _{m \rightarrow+\infty} \lim _{n \rightarrow+\infty} \sum_{i=1}^{N} \int_{\Omega}\left(a_{i}\left(x, T_{k}\left(u_{n}\right), \nabla T_{k}\left(u_{n}\right)\right)-a_{i}\left(x, T_{k}\left(u_{n}\right), \nabla T_{k}(u)\right)\right)$

$$
\partial_{i}\left(T_{k}\left(u_{n}\right)-T_{k}(u)\right)\left(1-h_{m}\left(u_{n}\right)\right) d x=0 .
$$

Let $\varphi=u_{n}+T_{k}\left(u_{n}\right)^{-}\left(1-h_{m}\left(u_{n}\right)\right)$ as test function in approximate problem (4.1), we obtain

$$
\begin{gathered}
-\sum_{i=1}^{N} \int_{\Omega} a_{i}\left(x, u_{n}, \nabla u_{n}\right) \partial_{i} T_{k}\left(u_{n}\right)^{-}\left(1-h_{m}\left(u_{n}\right)\right) d x+\sum_{i=1}^{N} \int_{\Omega} a_{i}\left(x, u_{n}, \nabla u_{n}\right) \partial_{i} u_{n} T_{k}\left(u_{n}\right)^{-} h_{m}^{\prime}\left(u_{n}\right) d x \\
-\sum_{i=1}^{N} \int_{\Omega} \phi_{i}\left(u_{n}\right) \partial_{i} T_{k}\left(u_{n}\right)^{-}\left(1-h_{m}\left(u_{n}\right)\right) d x+\sum_{i=1}^{N} \int_{\Omega} \phi_{i}^{n}\left(u_{n}\right) \partial_{i} u_{n} T_{k}\left(u_{n}\right)^{-} h_{m}^{\prime}\left(u_{n}\right) d x \\
\leq-\int_{\Omega} f_{n} T_{k}\left(u_{n}\right)^{-}\left(1-h_{m}\left(u_{n}\right)\right) d x
\end{gathered}
$$

By (4.12) and (4.13), we have $\lim _{m \rightarrow+\infty} \lim _{n \rightarrow+\infty} \sum_{i=1}^{N} \int_{\Omega} a_{i}\left(x, u_{n}, \nabla u_{n}\right) \partial_{i} u_{n} T_{k}\left(u_{n}\right)^{-} h_{m}^{\prime}\left(u_{n}\right) d x=0$. Then the second integral in (4.23) converges to zero as $n$ and $m$ tends to $+\infty$. Since $\partial_{i} T_{k}\left(u_{n}\right)^{-} \rightarrow \partial_{i} T_{k}(u)^{-}$in $L^{p_{i}}(\Omega)$ and $\phi_{i}\left(T_{k}\left(u_{n}\right)\right)(1-$ $\left.h_{m}\left(u_{n}\right)\right) \rightarrow \phi_{i}\left(T_{k}(u)\right)\left(1-h_{m}(u)\right)$ strongly in $L^{p_{i}^{\prime}}(\Omega)$, we have

$$
\lim _{m \rightarrow+\infty} \lim _{n \rightarrow+\infty} \sum_{i=1}^{N} \int_{\Omega} \phi_{i}\left(u_{n}\right) \partial_{i} T_{k}\left(u_{n}\right)^{-}\left(1-h_{m}\left(u_{n}\right)\right) d x=\lim _{m \rightarrow+\infty} \sum_{i=1}^{N} \int_{\Omega} \phi_{i}\left(T_{k}(u)\right) \partial_{i} T_{k}(u)^{-}\left(1-h_{m}(u)\right) d x .
$$

By Lebesgue's theorem, we get

$$
\lim _{m \rightarrow+\infty} \sum_{i=1}^{N} \int_{\Omega} \phi_{i}\left(T_{k}(u)\right) \partial_{i} T_{k}(u)^{-}\left(1-h_{m}(u)\right) d x=0 .
$$

Then the third integral in (4.23) converges to zero as $n$ and $m$ tends to $+\infty$.

We set $\Phi_{i}^{n}(t)=\int_{0}^{t} \phi_{i}(s) T_{k}(s)^{-} h_{m}^{\prime}(s) d s$, by Green's Formula, we have

$$
\sum_{i=1}^{N} \int_{\Omega} \phi_{i}^{n}\left(u_{n}\right) \partial_{i} u_{n} T_{k}\left(u_{n}\right)^{-} h_{m}^{\prime}\left(u_{n}\right) d x=\sum_{i=1}^{N} \int_{\Omega} \partial_{i} \Phi_{i}^{n}\left(u_{n}\right) d x=0 .
$$

Then the fourth integral in (4.23) converges to zero as $n$ and $m$ tend to $+\infty$. By the Lebesgue's theorem, we have the integral on the right hand in (4.23) converges to zero as $n$ and $m$ tend to $+\infty$. We deduce

$$
\lim _{m \rightarrow+\infty} \lim _{n \rightarrow+\infty} \sum_{i=1}^{N} \int_{\left\{u_{n} \leq 0\right\}} a_{i}\left(x, u_{n}, \nabla u_{n}\right) \partial_{i} T_{k}\left(u_{n}\right)\left(1-h_{m}\left(u_{n}\right)\right) d x=0 .
$$

Besides this, for $\eta$ small enough, we take $\varphi=u_{n}-\eta T_{k}\left(u_{n}^{+}-\psi^{+}\right)\left(1-h_{m}\left(u_{n}\right)\right)$ as test function in approximate problem (4.1), we obtain

$$
\begin{gathered}
\sum_{i=1}^{N} \int_{\Omega} a_{i}\left(x, u_{n}, \nabla u_{n}\right) \partial_{i} T_{k}\left(u_{n}^{+}-\psi^{+}\right)\left(1-h_{m}\left(u_{n}\right)\right) d x-\sum_{i=1}^{N} \int_{\Omega} a_{i}\left(x, u_{n}, \nabla u_{n}\right) \partial_{i} u_{n} T_{k}\left(u_{n}^{+}-\psi^{+}\right) h_{m}^{\prime}\left(u_{n}\right) d x \\
+\sum_{i=1}^{N} \int_{\Omega} \phi_{i}^{n}\left(u_{n}\right) \partial_{i} T_{k}\left(u_{n}^{+}-\psi^{+}\right)\left(1-h_{m}\left(u_{n}\right)\right) d x-\sum_{i=1}^{N} \int_{\Omega} \phi_{i}^{n}\left(u_{n}\right) \partial_{i} u_{n} T_{k}\left(u_{n}^{+}-\psi^{+}\right) h_{m}^{\prime}\left(u_{n}\right) d x
\end{gathered}
$$




$$
\leq \int_{\Omega} f_{n} T_{k}\left(u_{n}^{+}-\psi^{+}\right)\left(1-h_{m}\left(u_{n}\right)\right) d x
$$

By Hölder's inequality, (3.1), (4.12) and (4.13), we have

$$
\lim _{m \rightarrow+\infty} \lim _{n \rightarrow+\infty} \sum_{i=1}^{N} \int_{\Omega} \phi_{i}^{n}\left(u_{n}\right) \partial_{i} u_{n} T_{k}\left(u_{n}^{+}-\psi^{+}\right) h_{m}^{\prime}\left(u_{n}\right) d x=0 .
$$

Using young's inequality, we obtain

$$
\begin{gathered}
\sum_{i=1}^{N} \int_{\Omega} a_{i}\left(x, u_{n}, \nabla u_{n}\right) \partial_{i} T_{k}\left(u_{n}^{+}-\psi^{+}\right)\left(1-h_{m}\left(u_{n}\right)\right) d x \leq \\
\sum_{i=1}^{N} \int_{\left\{-(m+1) \leq u_{n} \leq-m\right\}} a_{i}\left(x, u_{n}, \nabla u_{n}\right) \partial_{i} u_{n} T_{k}\left(u_{n}^{+}-\psi^{+}\right) d x+\int_{\Omega} f_{n} T_{k}\left(u_{n}^{+}-\psi^{+}\right)\left(1-h_{m}\left(u_{n}\right)\right) d x \\
+\sum_{i=1}^{N} \int_{\left\{u_{n}^{+}-\psi^{+} \leq k\right\}} \phi_{i}^{n}\left(u_{n}\right) \partial_{i} u_{n}^{+}\left(1-h_{m}\left(u_{n}\right)\right) d x+\sum_{i=1}^{N} \int_{\left\{u_{n}^{+}-\psi^{+} \leq k\right\}} \phi_{i}^{n}\left(u_{n}\right) \partial_{i} \psi^{+}\left(1-h_{m}\left(u_{n}\right)\right) d x
\end{gathered}
$$

By (4.12), we have the first integral on the right hand converges to zero as $n$ and $m$ tend to $+\infty$. Using the Lebesque's theorem, we obtain the second integral in the right hand converges to zero as $n$ and $m$ tend to $+\infty$. Since

$$
\begin{gathered}
\sum_{i=1}^{N} \int_{\left\{u_{n}^{+}-\psi^{+} \leq k\right\}} \phi_{i}^{n}\left(u_{n}\right) \partial_{i} u_{n}^{+}\left(1-h_{m}\left(u_{n}\right)\right) d x \\
=\sum_{i=1}^{N} \int_{\Omega} \phi_{i}^{n}\left(T_{\left\{k+\left\|\psi^{+}\right\|_{L^{\infty}(\Omega)}\right\}}\left(u_{n}\right)\right) \partial_{i} T_{\left\{k+\left\|\psi^{+}\right\|_{L^{\infty}(\Omega)}\right\}}\left(u_{n}^{+}\right)\left(1-h_{m}\left(u_{n}\right)\right) d x .
\end{gathered}
$$

Since $\partial_{i} T_{\left\{k+\left\|\psi^{+}\right\|_{L^{\infty}(\Omega)}\right\}}\left(u_{n}^{+}\right) \rightarrow \partial_{i} T_{\left\{k+\left\|\psi^{+}\right\|_{L^{\infty}(\Omega)}\right.}\left(u^{+}\right)$weakly in $L^{p_{i}}(\Omega)$ and $\phi_{i}^{n}\left(T_{\left\{k+\left\|\psi^{+}\right\|_{L^{\infty}(\Omega)}\right\}}\left(u_{n}\right)\right)\left(1-h_{m}\left(u_{n}\right)\right) \rightarrow$ $\phi_{i}\left(T_{\left\{k+\left\|\psi^{+}\right\|_{L^{\infty}(\Omega)}\right.}(u)\right)\left(1-h_{m}\left(u_{n}\right)\right)$ strongly in $L^{p_{i}^{\prime}}(\Omega)$, we have

$$
\begin{aligned}
& \sum_{i=1}^{N} \int_{\Omega} \phi_{i}^{n}\left(T_{\left\{k+\left\|\psi^{+}\right\|_{L^{\infty}(\Omega)}\right\}}\left(u_{n}\right)\right) \partial_{i} T_{\left\{k+\left\|\psi^{+}\right\|_{L^{\infty}(\Omega)}\right\}}\left(u_{n}^{+}\right)\left(1-h_{m}\left(u_{n}\right)\right) d x \\
= & \sum_{i=1}^{N} \int_{\Omega} \phi_{i}\left(T_{\left\{k+\left\|\psi^{+}\right\|_{L^{\infty}(\Omega)}\right.}(u)\right) \partial_{i} T_{\left\{k+\left\|\psi^{+}\right\|_{L^{\infty}(\Omega)}\right\}}(u)\left(1-h_{m}(u)\right) d x+\varepsilon(n) .
\end{aligned}
$$

By Lebesgue's theorem, we have

$$
\lim _{m \rightarrow \infty} \sum_{i=1}^{N} \int_{\Omega} \phi_{i}\left(T_{\left\{k+\left\|\psi^{+}\right\|_{L^{\infty}(\Omega)}\right\}}(u)\right) \partial_{i} T_{\left\{k+\left\|\psi^{+}\right\|_{L^{\infty}(\Omega)}\right.}(u)\left(1-h_{m}(u)\right) d x=0 .
$$

Then, we have the third integral converges to zero as $n$ and $m$ tend to $+\infty$. Similarly as (4.24), we obtain

$$
\lim _{m \rightarrow+\infty} \lim _{n \rightarrow+\infty} \sum_{i=1}^{N} \int_{\left\{u_{n}>0\right\}} a_{i}\left(x, u_{n}, \nabla u_{n}\right) \partial_{i} T_{k}\left(u_{n}\right)\left(1-h_{m}\left(u_{n}\right)\right)=0 .
$$

Combining (4.24) and (4.27), we get

$$
\lim _{m \rightarrow+\infty} \lim _{n \rightarrow+\infty} \sum_{i=1}^{N} \int_{\Omega} a_{i}\left(x, u_{n}, \nabla u_{n}\right) \partial_{i} T_{k}\left(u_{n}\right)\left(1-h_{m}\left(u_{n}\right)\right) d x=0 .
$$

Furthermore, we have

$$
\sum_{i=1}^{N} \int_{\Omega}\left(a_{i}\left(x, T_{k}\left(u_{n}\right), \nabla T_{k}\left(u_{n}\right)\right)-a_{i}\left(x, T_{k}\left(u_{n}\right), \nabla T_{k}(u)\right)\right)\left(\partial_{i} T_{k}\left(u_{n}\right)-\partial_{i} T_{k}(u)\right) d x
$$




$$
\begin{gathered}
=\sum_{i=1}^{N} \int_{\Omega}\left(a_{i}\left(x, T_{k}\left(u_{n}\right), \nabla T_{k}\left(u_{n}\right)\right)-a_{i}\left(x, T_{k}\left(u_{n}\right), \nabla T_{k}(u)\right)\right)\left(\partial_{i} T_{k}\left(u_{n}\right)-\partial_{i} T_{k}(u)\right) h_{m}\left(u_{n}\right) d x \\
\quad+\sum_{i=1}^{N} \int_{\Omega}\left(a_{i}\left(x, T_{k}\left(u_{n}\right), \nabla T_{k}\left(u_{n}\right)\right)\right) \partial_{i} T_{k}\left(u_{n}\right)\left(1-h_{m}\left(u_{n}\right)\right) d x \\
\quad-\sum_{i=1}^{N} \int_{\Omega}\left(a_{i}\left(x, T_{k}\left(u_{n}\right), \nabla T_{k}\left(u_{n}\right)\right)\right) \partial_{i} T_{k}(u)\left(1-h_{m}\left(u_{n}\right)\right) d x \\
-\sum_{i=1}^{N} \int_{\Omega}\left(a_{i}\left(x, T_{k}\left(u_{n}\right), \nabla T_{k}(u)\right)\right)\left(\partial_{i} T_{k}\left(u_{n}\right)-\partial_{i} T_{k}(u)\right)\left(1-h_{m}\left(u_{n}\right)\right) d x .
\end{gathered}
$$

By (4.21) and (4.28), the first and the second integrals on the right hand side converge to zero as $n$ and $m$ tend to $+\infty$.

Since $\left(a_{i}\left(x, T_{k}\left(u_{n}\right), \nabla T_{k}\left(u_{n}\right)\right)\right)_{n}$ is bounded in $L^{p_{i}^{\prime}}(\Omega)$ and $\partial_{i} T_{k}(u)\left(1-h_{m}\left(u_{n}\right)\right)$ converge to zero in $L^{p_{i}}(\Omega)$ as $n$ and $m$ tend to $+\infty$, hence the third integral on the right hand side converge to zero as $n$ and $m$ tend to $+\infty$.

So, since $a_{i}\left(x, T_{k}\left(u_{n}\right), \nabla T_{k}\left(u_{n}\right)\right)\left(1-h_{m}\left(u_{n}\right)\right)$ converges to $a_{i}\left(x, T_{k}(u), \nabla T_{k}(u)\right)\left(1-h_{m}(u)\right)$ strongly in $L^{p_{i}^{\prime}}(\Omega)$ and $\partial_{i} T_{k}\left(u_{n}\right) \rightarrow \partial_{i} T_{k}(u)$ weakly in $L^{p_{i}}(\Omega)$, we obtain the fourth integral on the right hand side converge to zero as $n$ and $m$ tend to $+\infty$. Then, we get (4.10).

Using (4.9), (4.10) and Lemma 3.2, we obtain

$$
T_{k}\left(u_{n}\right) \rightarrow T_{k}(u) \text { strongly in } W_{0}^{1, \vec{p}}(\Omega) \text { and a. e. in } \Omega \quad \forall k>0 .
$$

Step4. Passing to the limit. Now, let $\varphi \in K_{\psi} \cap L^{\infty}(\Omega)$, we take $v=u_{n}-T_{k}\left(u_{n}-\varphi\right)$ as test function in approximate problem (4.1), we obtain

$$
\begin{gathered}
\sum_{i=1}^{N} \int_{\Omega} a_{i}\left(x, u_{n}, \nabla u_{n}\right) \partial_{i} T_{k}\left(u_{n}-\varphi\right) d x+\sum_{i=1}^{N} \int_{\Omega} \phi_{i}^{n}\left(u_{n}\right) \partial_{i} T_{k}\left(u_{n}-\varphi\right) d x \\
\leq \int_{\Omega} f_{n} T_{k}\left(u_{n}-\varphi\right) d x
\end{gathered}
$$

which implies that,

$$
\begin{gathered}
\sum_{i=1}^{N} \int_{\Omega} a_{i}\left(x, T_{k+\|\varphi\|_{\infty}}\left(u_{n}\right), \nabla T_{k+\|\varphi\|_{\infty}}\left(u_{n}\right)\right) \partial_{i} T_{k}\left(u_{n}-\varphi\right) d x+\sum_{i=1}^{N} \int_{\Omega} \phi_{i}\left(T_{k+\|\varphi\|_{\infty}}\left(u_{n}\right)\right) \partial_{i} T_{k}\left(u_{n}-\varphi\right) d x \\
\leq \int_{\Omega} f_{n} T_{k}\left(u_{n}-\varphi\right) d x
\end{gathered}
$$

Since $T_{k}\left(u_{n}\right) \rightarrow T_{k}(u)$ strongly in $W_{0}^{1, \vec{p}}(\Omega)$ and a. e. in $\Omega \quad \forall k>0$, we have $a_{i}\left(x, T_{k+\|\varphi\|_{\infty}}\left(u_{n}\right), \nabla T_{k+\|\varphi\|_{\infty}}\left(u_{n}\right)\right) \rightarrow a_{i}\left(x, T_{k+\|\varphi\|_{\infty}}(u), \nabla T_{k+\|\varphi\|_{\infty}}(u)\right)$ weakly in $L^{p_{i}^{\prime}}(\Omega)$, $\phi_{i}\left(T_{k+\|\varphi\|_{\infty}}\left(u_{n}\right)\right) \rightarrow \phi_{i}\left(T_{k+\|\varphi\|_{\infty}}(u)\right)$ strongly in $L^{p_{i}^{\prime}}(\Omega)$ and $\partial_{i} T_{k}\left(u_{n}-\varphi\right) \rightarrow \partial_{i} T_{k}(u-\varphi)$ strongly in $L^{p_{i}}(\Omega)$ we can pass to limit in

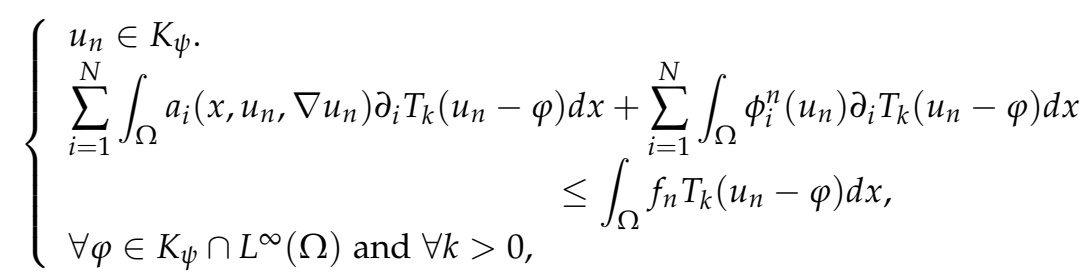

this completes the proof of theorem 4.1 . 


\section{Appendix}

In this section, we will show that the operator $B_{n}=A+\Phi_{n}$ is coercive and pseudo-monotone .

Proof of Lemma 4.1. We consider the operator $\Phi_{n}: K_{\psi} \rightarrow W^{-1, p^{\prime}}(\Omega)$ defined by $<\Phi_{n} u, v>=\sum_{i=1}^{N} \int_{\Omega} \phi_{i}^{n}(u) \partial_{i} v d x$. By Hölder's inequality, we have, for all $u, v \in X$,

$$
\begin{aligned}
\left|<\Phi_{n} u, v>\right| & \leq \sum_{i=1}^{N}\left(\int_{\Omega}\left|\phi_{i}\left(T_{n}(u)\right)\right|^{p_{i}^{\prime}} d x\right)^{\frac{1}{p_{i}^{\prime}}}\left(\int_{\Omega}\left|\partial_{i} v\right|^{p_{i}} d x\right)^{\frac{1}{p_{i}}} \\
& \leq \sum_{i=1}^{N}\left(\int_{\Omega} \sup _{|s| \leq n}\left|\phi_{i}(s)\right|^{p_{i}^{\prime}} d x\right)^{\frac{1}{p_{i}^{\prime}}}\left(\int_{\Omega}\left|\partial_{i} v\right|^{p_{i}} d x\right)^{\frac{1}{p_{i}}} \\
& \leq \sum_{i=1}^{N}\left(\int_{\Omega}\left(\sup _{|s| \leq n}\left|\phi_{i}(s)\right|+1\right)^{p_{i}^{\prime}} d x\right)^{\frac{1}{p_{i}^{\prime}}}\left(\int_{\Omega}\left|\partial_{i} v\right|^{p_{i}} d x\right)^{\frac{1}{p_{i}}} \\
& \leq \sum_{i=1}^{N}\left(\sup _{|s| \leq n}\left|\phi_{i}(s)\right|+1\right)\left(\int_{\Omega} 1 d x\right)^{\frac{1}{p_{i}^{\prime}}}\left(\int_{\Omega}\left|\partial_{i} v\right|^{p_{i}} d x\right)^{\frac{1}{p_{i}}} \\
& \leq \max _{1 \leq i \leq N}\left(\sup _{|s| \leq n}\left|\phi_{i}(s)\right|+1\right)(\text { meas }(\Omega)+1)^{\frac{1}{p_{-}^{\prime}}} \sum_{i=1}^{N}\left(\int_{\Omega}\left|\partial_{i} v\right|^{p_{i}} d x\right)^{\frac{1}{p_{i}}} \\
& \leq C(n)\|v\|_{W_{0}^{1, \vec{p}}(\Omega)^{\prime}}
\end{aligned}
$$

which implies that $\frac{\left|\left\langle\Phi_{n} u, v\right\rangle\right|}{\|v\|_{1, \vec{p}}} \leq C(n)$. Moreover, let $v_{0} \in K_{\psi}$, thanks to Hölder's inequality and (3.2), we have

$$
\begin{aligned}
\left|<A v, v_{0}>\right| & \leq \sum_{i=1}^{N}\left(\int_{\Omega}\left|a_{i}(x, v, \nabla v)\right|^{p_{i}^{\prime}} d x\right)^{\frac{1}{p_{i}^{\prime}}}\left(\int_{\Omega}\left|\partial_{i} v_{0}\right|^{p_{i}} d x\right)^{\frac{1}{p_{i}}} \\
& \leq \beta \sum_{i=1}^{N}\left(\int_{\Omega}\left(j_{i}(x)^{p_{i}^{\prime}}+|v|^{p_{i}}+\left|\partial_{i} v\right|^{p_{i}}\right) d x\right)^{\frac{1}{p_{i}^{\prime}}}\left(\int_{\Omega}\left|\partial_{i} v_{0}\right|^{p_{i}} d x\right)^{\frac{1}{p_{i}}} \\
& \left.\leq \beta \sum_{i=1}^{N}\left(C_{1}+\int_{\Omega}\left|\partial_{i} v\right|^{p_{i}}+\int_{\Omega}\left|\partial_{i} v\right|^{p_{i}}\right) d x\right)^{\frac{1}{p_{i}^{\prime}}}\left(\int_{\Omega}\left|\partial_{i} v_{0}\right|^{p_{i}} d x\right)^{\frac{1}{p_{i}}} \\
& \left.\leq \beta \sum_{i=1}^{N} C_{1}^{\frac{1}{p_{i}^{\prime}}}\left(1+\frac{2}{C_{1}} \sum_{i=1}^{N} \int_{\Omega}\left|\partial_{i} v\right|^{p_{i}}\right) d x\right)^{\frac{1}{p_{i}^{\prime}}}\left(\int_{\Omega}\left|\partial_{i} v_{0}\right|^{p_{i}} d x\right)^{\frac{1}{p_{i}}} \\
& \left.\leq \beta C_{2} \sum_{i=1}^{N}\left(1+\frac{2}{C_{1}} \sum_{i=1}^{N} \int_{\Omega}\left|\partial_{i} v\right|^{p_{i}}\right) d x\right)^{\frac{1}{p_{-}^{\prime}}}\left(\int_{\Omega}\left|\partial_{i} v_{0}\right|^{p_{i}} d x\right)^{\frac{1}{p_{i}}} \\
& \left.\leq \beta C_{2} \sum_{i=1}^{N}\left(1+C_{3}\left(\sum_{i=1}^{N} \int_{\Omega}\left|\partial_{i} v\right|^{p_{i}}\right) d x\right)^{\frac{1}{p_{-}^{\prime}}}\right)\left(\int_{\Omega}\left|\partial_{i} v_{0}\right|^{p_{i}} d x\right)^{\frac{1}{p_{i}}} \\
& \left.\leq \beta C_{2}\left(1+C_{3}\left(\sum_{i=1}^{N} \int_{\Omega}\left|\partial_{i} v\right|^{p_{i}}\right) d x\right)^{\frac{1}{p_{-}^{\prime}}}\right) \sum_{i=1}^{N}\left(\int_{\Omega}\left|\partial_{i} v_{0}\right|^{p_{i}} d x\right)^{\frac{1}{p_{i}}} \\
& \left.\leq \beta C_{2}\left(1+C_{3}\left(\sum_{i=1}^{N} \int_{\Omega}\left|\partial_{i} v\right|^{p_{i}}\right) d x\right)^{\frac{1}{p_{-}^{\prime}}}\right)\left\|v_{0}\right\|_{W_{0}^{1,} \vec{p}}(\Omega)
\end{aligned}
$$

Hence

$$
\frac{\left|<A v, v-v_{0}>\right|}{\|v\|_{W_{0}^{1, \vec{p}}(\Omega)}} \geq \alpha \frac{\sum_{i=1}^{N} \int_{\Omega}\left|\partial_{i} v\right|^{p_{i}} d x}{\|v\|_{W_{0}^{1, \vec{p}}(\Omega)}}-\frac{\beta C_{2}\left\|v_{0}\right\|_{W_{0}^{1, \vec{p}}(\Omega)}}{\|v\|_{W_{0}^{1, \vec{p}}(\Omega)}}
$$


Then

$$
-\frac{\beta C_{2} C_{3}}{\|v\|_{W_{0}^{1, \vec{p}}(\Omega)}}\left(\sum_{i=1}^{N} \int_{\Omega}\left|\partial_{i} v\right|^{p_{i}} d x\right)^{\frac{1}{p_{-}^{\prime}}}\left\|v_{0}\right\|_{W_{0}^{1, \vec{p}}(\Omega)} .
$$

$$
\begin{aligned}
& \frac{\left|<A v, v-v_{0}>\right|}{\|v\|_{W_{0}^{1, \vec{p}}(\Omega)}} \geq \\
& \alpha \frac{\sum_{i=1}^{N} \int_{\Omega}\left|\partial_{i} v\right|^{p_{i}} d x}{\|v\|_{W_{0}^{1, \vec{p}}(\Omega)}}\left[1-\frac{\beta}{\alpha} C_{2} C_{3}\left(\sum_{i=1}^{N} \int_{\Omega}\left|\partial_{i} v\right|^{p_{i}} d x\right)^{\frac{1}{p_{-}^{\prime}}-1}\left\|v_{0}\right\|_{W_{0}^{1, \vec{p}}(\Omega)}\right]-\frac{\beta C_{2}\left\|v_{0}\right\|_{W_{0}^{1, \vec{p}}(\Omega)}}{\|v\|_{W_{0}^{1, \vec{p}}(\Omega)}} .
\end{aligned}
$$

Additionally, by Jensen's inequality, we have

where

$$
\begin{aligned}
\|v\|_{1, \vec{p}}^{p_{-}^{+}} & =\left(\sum_{i=1}^{N}\left(\int_{\Omega}\left|\partial_{i} v\right|^{p_{i}} d x\right)^{\frac{1}{p_{i}}}\right)^{p_{-}^{+}} \\
\leq & \left(\sum_{i=1}^{N}\left(\int_{\Omega}\left|\partial_{i} v\right|^{p_{i}} d x\right)^{\frac{1}{p_{-}^{+}}}\right)^{p_{-}^{+}} \\
\leq C & \sum_{i=1}^{N} \int_{\Omega}\left|\partial_{i} v\right|^{p_{i}} d x,
\end{aligned}
$$

$$
p_{-}^{+}= \begin{cases}p^{-} & \text {if }\left\|\partial_{i} v\right\|_{L^{p_{i}(\Omega)}} \geq 1 \\ p^{+} & \text {if }\left\|\partial_{i} v\right\|_{L^{p_{i}(\Omega)}}<1\end{cases}
$$

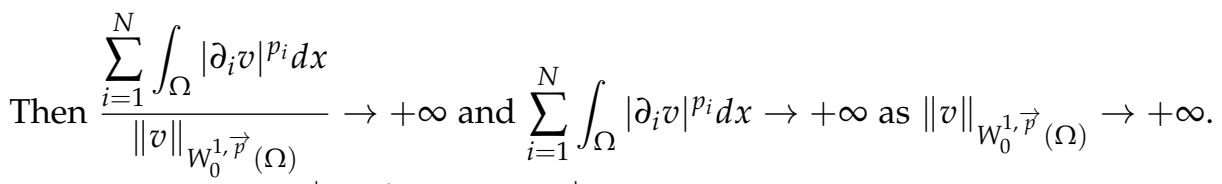

Using (5.1), we get $\frac{\left|\left\langle A v, v-v_{0}\right\rangle\right|}{\|v\|_{W_{0}^{1, \vec{p}}(\Omega)}} \rightarrow+\infty$ as $\|v\|_{1, \vec{p}} \rightarrow+\infty$.

Since $\frac{\left\langle\Phi_{n} v, v\right\rangle}{\|v\|_{W_{0}^{1, \vec{p}}(\Omega)}}$ and $\frac{\left\langle\Phi_{n} v, v_{0}\right\rangle}{\|v\|_{W_{0}^{1, \vec{p}}(\Omega)}}$ are bounded, then we have

$\frac{\left\langle B_{n} v, v-v_{0}\right\rangle}{\|v\|_{W_{0}^{1, \vec{p}}(\Omega)}}=\frac{\left\langle A v, v-v_{0}\right\rangle}{\|v\|_{W_{0}^{1, \vec{p}}(\Omega)}}+\frac{\left\langle\Phi_{n} v, v-v_{0}\right\rangle}{\|v\|_{W_{0}^{1, \vec{p}}(\Omega)}} \rightarrow+\infty$ as $\|v\|_{W_{0}^{1, \vec{p}}(\Omega)} \rightarrow+\infty$. We deduce that the operator $B_{n}=A+\Phi_{n}$ is coercive. It remains to prove that the operator $B_{n}$ is pseudo-monotone. Let $\left(u_{k}\right)_{k}$ be a sequence in $W_{0}^{1, \vec{p}}(\Omega)$ such that

$$
\left\{\begin{array}{l}
u_{k} \rightarrow u \quad \text { weakly in } W_{0}^{1, \vec{p}}(\Omega) \\
B_{n} u_{k} \rightarrow \chi \quad \text { weakly in } W^{-1, \overrightarrow{p^{\prime}}}(\Omega) \\
\limsup _{k \rightarrow+\infty}<B_{n} u_{k}, u_{k}>\leq<\chi, u>.
\end{array}\right.
$$

We will prove that $\chi=B_{n} u$ and $<B_{n} u_{k}, u_{k}>\rightarrow<\chi, u>$ as $k \rightarrow+\infty$. Since $W_{0}^{1, \vec{p}}(\Omega) \hookrightarrow L^{p^{-}}(\Omega)$, then $u_{k} \rightarrow u$ strongly in $L^{p^{-}}(\Omega)$ and a.e. in $\Omega$ for a subsequence denoted again $\left(u_{k}\right)_{k}$. Since $\left(u_{k}\right)_{k}$ is bounded in $W_{0}^{1, \vec{p}}(\Omega)$, by (3.2), we have $\left(a_{i}\left(x, u_{k}, \nabla u_{k}\right)\right)_{k}$ is bounded in $L^{p_{i}^{\prime}}(\Omega)$. Then there exists a function $\varphi_{i} \in L^{p_{i}^{\prime}}(\Omega)$ such that

$$
a_{i}\left(x, u_{k}, \nabla u_{k}\right) \rightarrow \varphi_{i} \text { as } k \rightarrow+\infty .
$$

What is more, since $\left(\phi_{i}^{n}\left(u_{k}\right)\right)_{k}$ is bounded in $L^{p_{i}^{\prime}}(\Omega)$ and $\phi_{i}^{n}\left(u_{k}\right) \rightarrow \phi_{i}^{n}(u)$ a.e. in $\Omega$, we have

$$
\phi_{i}^{n}\left(u_{k}\right) \rightarrow \phi_{i}^{n}(u) \text { strongly in } L^{p_{i}^{\prime}}(\Omega) \text { as } k \rightarrow+\infty .
$$


For all $v \in W_{0}^{1, \vec{p}}(\Omega)$, using (5.2) and (5.3), we obtain

$\langle\chi, v\rangle=\lim _{k \rightarrow+\infty}\left\langle B_{n} u_{k}, v\right\rangle$

$$
\begin{aligned}
& =\lim _{k \rightarrow+\infty} \sum_{i=1}^{N} \int_{\Omega} a_{i}\left(x, u_{k}, \nabla u_{k}\right) \partial_{i} v d x+\lim _{k \rightarrow+\infty} \sum_{i=1}^{N} \int_{\Omega} \phi_{i}^{n}\left(u_{k}\right) \partial_{i} v d x \\
& =\sum_{i=1}^{N} \int_{\Omega} \varphi_{i} \partial_{i} v d x+\sum_{i=1}^{N} \int_{\Omega} \phi_{i}^{n}(u) \partial_{i} v d x .
\end{aligned}
$$

Hence, we have

$$
\begin{aligned}
& \limsup _{k \rightarrow+\infty}<B_{n} u_{k}, u_{k}>=\limsup _{k \rightarrow+\infty}\left[\sum_{i=1}^{N} \int_{\Omega} a_{i}\left(x, u_{k}, \nabla u_{k}\right) \partial_{i} u_{k} d x+\sum_{i=1}^{N} \int_{\Omega} \phi_{i}^{n}\left(u_{k}\right) \partial_{i} u_{k} d x\right] \\
& =\limsup _{k \rightarrow+\infty} \sum_{i=1}^{N} \int_{\Omega} a_{i}\left(x, u_{k}, \nabla u_{k}\right) \partial_{i} u_{k} d x+\sum_{i=1}^{N} \int_{\Omega} \phi_{i}^{n}(u) \partial_{i} u d x \\
& \leq\langle\chi, u\rangle \\
& =\sum_{i=1}^{N} \int_{\Omega} \varphi_{i} \partial_{i} u d x+\sum_{i=1}^{N} \int_{\Omega} \phi_{i}^{n}(u) \partial_{i} u d x
\end{aligned}
$$

which implies that

$$
\limsup _{k \rightarrow+\infty} \sum_{i=1}^{N} \int_{\Omega} a_{i}\left(x, u_{k}, \nabla u_{k}\right) \partial_{i} u_{k} d x \leq \sum_{i=1}^{N} \int_{\Omega} \varphi_{i} \partial_{i} u d x
$$

By (3.3), we have $\sum_{i=1}^{N} \int_{\Omega}\left(a_{i}\left(x, u_{k}, \nabla u_{k}\right)-a_{i}\left(x, u_{k}, \nabla u\right)\right)\left(\partial_{i} u_{k}-\partial_{i} u\right) d x>0$.

$$
\begin{aligned}
& \text { Then } \\
& \begin{aligned}
\sum_{i=1}^{N} \int_{\Omega} a_{i}\left(x, u_{k}, \nabla u_{k}\right) \partial_{i} u_{k} d x \geq- & \sum_{i=1}^{N} \int_{\Omega} a_{i}\left(x, u_{k}, \nabla u\right) \partial_{i} u d x+\sum_{i=1}^{N} \int_{\Omega} a_{i}\left(x, u_{k}, \nabla u_{k}\right) \partial_{i} u d x . \\
& +\sum_{i=1}^{N} \int_{\Omega} a_{i}\left(x, u_{k}, \nabla u\right) \partial_{i} u_{k} d x .
\end{aligned}
\end{aligned}
$$

Using (5.2), we get

$$
\liminf _{k \rightarrow+\infty} \sum_{i=1}^{N} \int_{\Omega} a_{i}\left(x, u_{k}, \nabla u_{k}\right) \partial_{i} u_{k} d x \geq \sum_{i=1}^{N} \int_{\Omega} \varphi_{i} \partial_{i} u d x
$$

Combining (5.4) and (5.5), we obtain

$$
\begin{aligned}
\lim _{k \rightarrow+\infty} \sum_{i=1}^{N} \int_{\Omega} a_{i}\left(x, u_{k}, \nabla u_{k}\right) \partial_{i} u_{k} d x=\sum_{i=1}^{N} \int_{\Omega} \varphi_{i} \partial_{i} u d x . \\
\lim _{k \rightarrow+\infty}<B_{n} u_{k}, u_{k}>=\lim _{k \rightarrow+\infty} \sum_{i=1}^{N} \int_{\Omega} a_{i}\left(x, u_{k}, \nabla u_{k}\right) \partial_{i} u_{k} d x+\lim _{k \rightarrow+\infty} \sum_{i=1}^{N} \int_{\Omega} \phi_{i}^{n}\left(u_{k}\right) \partial_{i} u_{k} d x \\
=\sum_{i=1}^{N} \int_{\Omega} \varphi_{i} \partial_{i} u d x+\sum_{i=1}^{N} \int_{\Omega} \phi_{i}^{n}(u) \partial_{i} u d x \\
=<\chi, u>.
\end{aligned}
$$

In addition to this, since $a_{i}\left(x, u_{k}, \nabla u\right)$ converges to $a_{i}(x, u, \nabla u)$ strongly in $L^{p_{i}^{\prime}}(\Omega)$, by (5.6), we obtain

$$
\sum_{i=1}^{N} \int_{\Omega}\left(a_{i}\left(x, u_{k}, \nabla u_{k}\right)-a_{i}\left(x, u_{k}, \nabla u\right)\right)\left(\partial_{i} u_{k}-\partial_{i} u\right) d x=0 .
$$

Using lemma 3.2, we get $u_{k}$ converges to $u$ strongly in $W_{0}^{1, \vec{p}}(\Omega)$ and a. e. in $\Omega$, then $a_{i}\left(x, u_{k}, \nabla u\right)$ converges to $a_{i}(x, u, \nabla u)$ weakly in $L^{p_{i}^{\prime}}(\Omega)$ and $\phi_{i}^{n}\left(u_{k}\right)$ converges to $\phi_{i}^{n}(u)$ strongly in $L^{p_{i}^{\prime}}(\Omega)$. Then for all $v \in W_{0}^{1, \vec{p}}(\Omega)$, we have 


$$
\begin{aligned}
<\chi, v> & =\lim _{k \rightarrow+\infty}\left\langle B_{n} u_{k}, v>\right. \\
& =\lim _{k \rightarrow+\infty} \sum_{i=1}^{N} \int_{\Omega} a_{i}\left(x, u_{k}, \nabla u_{k}\right) \partial_{i} v d x+\lim _{k \rightarrow+\infty} \sum_{i=1}^{N} \int_{\Omega} \phi_{i}\left(u_{k}\right) \partial_{i} v d x \\
& =\sum_{i=1}^{N} \int_{\Omega} a_{i}(x, u, \nabla u) \partial_{i} v d x+\sum_{i=1}^{N} \int_{\Omega} \phi_{i}(u) \partial_{i} v d x \\
& =<B_{n} u, v>
\end{aligned}
$$

which implies that $B_{n} u=\chi$.

\section{References}

[1] Aharouch L, Akdim Y. Strongly Nonlinear Elliptic Unilateral Problems without sign condition and $\mathrm{L}^{1}$ Data. Appl. Anal. (2005); 11-31.

[2] Akdim Y, Azroul E, Benkirane A. Existence Results for Quasilinear Degenerated Equations Via Strong Convergence of Truncations. Rev. Mat. Complut., (2001) 17; 359-379.

[3] Akdim Y, Benkirane A, El Moumni M. Existence results for nonlinear elliptic problems with lower order terms. Int. J. Evol. Equ., (2013) 8; 4: 257-276.

[4] Antontsev S, Chipot M. Anisotropic equations: uniqueness and existence results. Differential Integral Equations (2008) 6; 401-419.

[5] Bendahmane M, Karlsen KH. Anisotropic nonlinear elliptic systems with measure data and anisotropic harmonic maps into spheres. Electronic. J. Differential Equations, ( 2006) 46; 1-30.

[6] Benkirane A, Bennouna J. Existence of entropy solutions for nonlinear problems in Orlicz spaces. Abs. and Apl. Ann., (2002) 7; 85-102.

[7] Benkirane A, Bennouna J. Existence and uniqueness of solution of unilateral problems with L1-data in Orlicz spaces. Ital. J. Pure Appl. Math., (2004) 16; 87-102.

[8] Bénilan P, Boccardo L, Gallouët T, Gariepy R, Pierre M, Vázquez J. An $L^{1}$-theory of existence and uniqueness of nonlinear elliptic equations. Ann. Sc. Norm. Sup. Pisa, CL. IV(1995).; 22 : 240-273.

[9] Boccardo L, Some nonlinear Dirichlet problems in $L^{1}$ involving lower order terms in divergence form. Progress in elliptic and parabolic partial differential equations (Capri, 1994), Pitman Res. Notes Math. Ser., 350, p. 43-57, Longman, Harlow, 1996.

[10] Boccardo L, Gallouët T. Strongly nonlinear elliptic equations having natural growth terms and $L^{1}$ data. Nonlinear Anal. T.M.A. (1992); 19: 573-578.

[11] Boccardo L, Gallouët T, Marcellini P. Anisotropic equations in L1 . Differential Integral Equations (1996) 1; 209212.

[12] Boccardo L, Gallouët T, Orsina L. Existence and nonexistence of solutions for some nonlinear elliptic equations. J. Anal. Math. (1997) 73; 203-223.

[13] Boccardo L, Murat F, Puel J.-P. Existence of bounded solution for nonlinear elliptic unilateral problems. Ann. Mat. Pura Appl. (1988) 152; 183-196.

[14] Di Castro A. Existence and regularity results for anisotropic elliptic problems. Adv. Nonlinear Stud. (2009)9; 367393.

[15] Di Castro A. Anisotropic elliptic problems wih natural growth terms. Manuscripta Math. (2011) 135: 521-543 .

[16] Di Nardo R, Feo F. Existence and uniqueness for nonlinear anisotropic elliptic equations. Arch. Math.(2014) 102; 141-153.

[17] Fragala I, Gazzola F, Kawohl B. Existence and nonexistence results for anisotropic quasilinear elliptic equations. Ann. Inst. H. Poincar Anal. Non Linéaire (2004) 5; 715-734.

[18] Guib O, Mercaldo A. Uniqueness results for noncoercive nonlinear elliptic equations with two lower order terms. Commun. Pure Appl. Anal. (2008) 1; 163-192.

[19] Leray J., Lions L. Quelques Mthodes de Rsolution des Problmes aux Limites Non linaires. Dunod, Paris, 1968.

[20] J. P. Gossez and V. Mustonen, Variational inequalities in Orlicz-Sobolev spaces, Nonlinear Anal. (1987) 11; $379-492$.

[21] Li FQ. Anisotropic elliptic equations in $L^{m}$. J. Convex Anal. (2001) 2; 417-422. 
[22] M.Troisi. Teoremi di inclusione per spazi di Sobolev non isotropi. Ricerche Mat (1969) 18; 3-24.

[23] Yazough C, Azroul E, Redwane H. Existence of solutions for some nonlinear elliptic unilateral problems with measure data. E. J.Q. Theo. of Di. Equations. (2013) 43; 1-21. 